\title{
LA MATERIA PRIMERA DE ENRIQUE DE GANTE VISTA POR DUNS ESCOTO
}

\author{
Antonio Pérez-Estévez \\ Universidad del Zulia, Venezuela
}

\section{RESUMEN}

Este trabajo demuestra cómo Duns Escoto no comprendió correctamente la doctrina que Enrique de Gante había elaborado sobre la materia prima. En efecto, de los tres seres que el Doctor Sólido le atribuye a la materia prima - el ser como criatura, el ser propio de su esencia y el ser en acto recibido de la forma-Duns Escoto solo descubre los dos últimos, a saber, el ser esencial y el ser en acto formal. Además hace decir a Enrique de Gante que la materia puede existir sola, sin forma, porque recibe directamente de Dios el ser subsistente per se que no es análogo al ser en acto formal. Existiría o sería en acto sin poseer el ser en acto, lo que es contradictorio.

Palabras clave: Duns Scoto., Enrique de Gante, Materia prima

\begin{abstract}
This essay proves that Duns Scotus did not understand rightly Henry of Ghent's doctrine on prime matter. In fact, of the three beings Doctor Solidus gives to prime matter - the being as creature, the essential being and the being in act received from form-, Duns Scotus discovers only the two last ones, that is, the essential being and the formal being in act. Besides, He makes Henry of Ghent saying that prime matter can exist alone, without form, due to the fact that it receives directly from God the subsistent being per se, a being that is not analogous to the being in act of form. In that case, prime matter would exist or would be in act without owning the being in act, what is contradictory.
\end{abstract}

Key words: Duns Scotus, Henry of Ghent, Prime matter

\section{INTRODUCCIÓN: DOCTRINAS DE ENRIQUE DE GANTE Y DUNS ESCOTO SOBRE LA MATERIA PRIMA}

Juan Duns Escoto escribe sus obras fundamentales algunos años más tarde de la muerte de Enrique de Gante ocurrida en 1293: la Lectura pertenece a los años finales del siglo XIII (1298-1299), la corrección de la Ordinatio se extiende desde 1300 hasta su muerte en 1308, los Reportata Parisiensia se ubican en 1305 y la redacción de las Quaestiones super libros Metaphysicorum Aristotelis se iniciaron antes del 1300 pero sus últimos libros pertenecen sin duda ya a los inicios del siglo XIV. Las dos obras que conservamos de Enrique de Gante, por otro lado - Quodlibeta y Summa Quaestionum ordinariorum - habían sido escritas en el lapso de tiempo en el que fue regente de la cátedra en la Universidad de Paris y que está comprendido entre los años 1276 y 1292. Los Quodlibeta fueron redactados a lo largo de 15 años: el Quodlibet $I$ en el que desarrolla por primera vez y con mayor precisión su doctrina sobre la materia prima fue escrito a finales de 1277 mientras que el Quodlibet $X V$ es de finales de 1292. Estos 
hechos significan que Duns Escoto pudo conocer los escritos de Enrique de Gante, personaje importante en los medios académicos de su tiempo pues fue llamado a formar parte de la comisión que redactó la condenación doctrinal del Obispo Tempier de 1277. De hecho, Duns Escoto debió haberlos leído con cuidado pues sus referencias y críticas a ellos son frecuentes. En este trabajo nos detendremos a analizar la visión que el franciscano tuvo de la doctrina que Enrique de Gante había elaborado en torno a la materia prima.

La doctrina sobre la materia prima que ambos desarrollaron encierra muchas analogías. Se contrapone a la de Tomás de Aquino y a la de su discípulo Egidio Romano, quienes sostenían que la materia prima, incognoscible en sí misma y cognoscible sólo por analogía, era pura potencia para recibir formas. Al conocimiento de la materia prima llegamos por analogía o comparación en los procesos de mutación sustancial o generación y corrupción, tal como había apuntado ya Aristóteles. Todo cambio sustancial implica una sustitución de formas sustanciales: una forma sustancial X desaparece y aparece una nueva forma sustancial Y. Pero para que ese cambio sustancial pueda producirse, hay que suponer que existe algo común que sirva de base o fundamento para tal cambio de formas sustanciales. A ese algo común, informe en sí mismo e ingenerable e incorruptible, sobre el que se da tal cambio de formas sustanciales, lo llamamos materia prima. Y la materia prima, en sí misma informe, no puede poseer el acto de ser, porque éste proviene siempre de la forma. Para Tomás de Aquino la esfera del ser queda reducida a ser en acto. Pero ser en acto tiene, para el Aquinate, un doble sentido: ser en acto por el que la forma produce la sustancia individual y ser en acto por el que la sustancia individual existe fuera de sus causas. Nada puede existir sin tener forma por lo que el acto de existir sólo es recibido por la sustancia individual, cuyo ser sustancial proviene de la forma. Ser en acto una sustancia equivale a tener forma, pues la forma es aquello por lo que algo es en acto, nam per formam ununquodque est in actu, o, tal como afirma en otro texto es lo mismo decir ser en acto que forma, ipsum autem quod est actus est forma ${ }^{1}$. Si ser (existir) equivale a ser en acto fuera de sus causas y ser en acto equivale a la forma, la materia prima no-es, debido a que no es en acto y a que no es una forma. De ahí que Tomás de Aquino diga de la materia prima que no pertenece al ámbito de los entes, es decir, diga que es un no-ente, sin que llegue a ser la nada absoluta. La materia prima, para el Aquinate y para Egidio Romano, queda reducida a ser pura potencia o capacidad de recibir formas sustanciales ${ }^{2}$.

Contra esta entidad extraña de la materia prima, que sin llegar a ser un ente - es un noente- no es tampoco la nada, van a elaborar su concepto de materia prima tanto Enrique de Gante como Juan Duns Escoto. De hecho, ambos van a partir de los mismos supuestos: a) que si se acepta el hilemorfismo, es decir, si se acepta que la materia es uno de los dos principios y una de las dos causas que constituyen una sustancia compuesta, tiene que ser un ente realmente distinto de la forma; b) que la entidad de la materia, como principio real de la sustancia individual compuesta, es mayor que la entidad de cualquier accidente. Enrique de Gante destaca que, si bien la materia es casi nada, porque su entidad se encuentra entre el ser en acto

1 Thomae Aquinatis, In Secundum de Anima, Marietti, Turín, 1955, $\mathrm{n}^{\circ}$ 319: Illud est causa alicuius ut substantia, id est forma, quod est causa essendi. Nam per formam unumquodque est actus. También, Summa theologi$c a$, Edición Leonina, $\mathrm{I}^{\mathrm{a}}$, q. 66, a. 1,c: ipsum autem est actus est forma. Dicere igitur materiam praecedere esse forma, est dicere ens actu sine actu, quod implicat contradictionem.

2 Thomae Aquinatis, Summa theologica, Edición leonina, Ia , q. 77, a. 1, ad 2um: Et ideo potentia materiae non est aliud quam eius essentia. También en Summa theologica, $\mathrm{I}^{\mathrm{a}}$, q. 115, a. 1, ad 2um: sed hoc est materia prima, quae est pura potentia.Aegidi Romani In Secundum Librum Sententiarum Quaestiones, Venecia, 1631, q. 2, art. 1, p. 13: intelligimus enim hic per materiam aliquid quod de se nullum actum habet... est ergo materia ipsa potentia pura...materia de se nec esse nec aliquem actum importat, quia si dicere de se aliquid esse vel aliquem actum, non esset potentia pura et posset de se intelligi vel cognosci. Dicere ergo materiam esse principium activum est dicere opposita, videlicet quod aliquid habeat esse et non esse, quod sit in potentia et non in potentia, quod sit in actu et non in actu. 
perfecto bajo la forma y el no-ente, sin embargo en su naturaleza es algo ${ }^{3}$. También Duns Escoto afirma que la materia, como sujeto del cambio sustancial, tiene que ser algo. Para ambos tiene que ser un ente, una esencia con un ser propio. Y si es un ente con una esencia y un ser propio, posee una idea en la mente divina y es, en sí misma, cognoscible aunque no lo sea para nuestro entendimiento, limitado a conocer a partir de lo que afecta a nuestros sentidos y deteriorado a causa del pecado original.

Enrique de Gante afirma textualmente que:

(la materia) en su naturaleza es algo... es un id quod est hecho y creado por Dios...y, aunque la hizo subordinada a la forma de manera que existieran la materia y la forma simultáneamente en el tiempo, en su origen y por naturaleza el ser y la creación de la materia es anterior a la forma ${ }^{4}$.

La materia prima es un $i d$ quod est, una esencia hecha y creada por Dios para existir subordinada a la forma y simultáneamente con ella en el tiempo. Pero en su origen y por naturaleza el ser de la materia es anterior al ser de la forma. Añadirá que la materia prima «es algo sustancial capaz de recibir la forma (del compuesto)», es decir, es algo con consistencia suficiente para poder existir por sí mismo, lo que supone que su ser encierra una entidad mayor que la de cualquier accidente 5 . Por eso, podrá por una acción especial de Dios existir sola sin forma alguna ${ }^{6}$.

Tampoco la materia prima es pura potencia para Duns Escoto. Como sujeto de todo cambio sustancial, la materia prima es algo ingenerable e incorruptible que permanece y sobre la que se produce la corrupción de una forma sustancial y la generación de una nueva forma sustancial. La materia prima como sujeto del cambio sustancial, permanece para formar parte de la nueva sustancia que nace como efecto de ese cambio sustancial. Por eso, Duns Escoto afirmará que

Se dice que la materia prima es principio per se y también causa per se; se dice también que es per se parte de lo engendrado, es también sujeto de la mutación... es también algo que permanece bajo cada uno de los términos de la mutación y de la generación y que está en potencia hacia la forma y se añade — según los teólogos- que es algo en lo que termina la creación?

La materia, expresa Duns Escoto, es un ser en potencia, entendiendo que la materia prima es un sujeto en potencia o un sujeto en el que hay la potencia de recibir formas sustanciales con las que constituirá sustancias individuales. La materia, como ser en potencia, es decir, como sujeto en el que se da la potencia de recibir nuevas formas sustanciales, tiene esencia propia y ser en acto propio, en cuanto que es fuera de sus causas, pero este ser en acto es sumamente

3 Henricus de Gadavo, Quodlibet I, Edición crítica de Raymond Macken, Leuven University Press, 1979, art. 10, p. 64, vv. 41-44: Et quamquam materia ita sit prope nihil, quod sit media inter ens actu perfectum sub forma et non ens, ipsa tamen in sua natura aliquid est...

4 Henricus de Gandavo, Quodlibet I, art. 10, p. 64, vv.42-50: in sua natura aliquid est...et id quod est a Deo factum atque creatum est..et licet fecit eam sub forma ut simul tempore fuerint semper materia et forma, origine tamen et natura prius est esse et creatio materiae quam forma in ipsa.

5 Quodlibet I, art. 10, p. 66, vv. 81-89: materia est aliqua natura et substantia quae est capax formarum, differens per essentiam a forma... ipsa est susceptibilis esse per se tamquam per se creabile et propriam habens ideam in mente divina.

6 O.c., art. 10, p. 71, vv. 97-01.

7 Ioannes Duns Scotus, Lectura II, dist. XII, q. única, n² 29 (Opera Omnia, Edición Vaticana, t. XIX, p. 7980): materia dicitur esse principium per se, et etiam per se causa; dicitur etiam per se esse pars geniti, est etiam subjectum mutationis... est etiam aliquid manens sub utroque terminorum mutationis et generationis in potentia ad formam, et additur - secundum theologos - quod est aliquid terminans creationem. 
imperfecto y, por eso, máximamente receptivo. Al tener esencia y ser en acto propios, la materia es en sí misma cognoscible, aunque no lo sea para nuestro entendimiento limitado, debido a su endeblez óntica.. El ser en acto mínimo de la materia es un ser sustancial, por lo que Duns Escoto dice que «nada hay tan cercano a la nada en el género de sustancia como la materia» ${ }^{8}$. Por ser sustancia y tener la consistencia óntica para existir en sí misma, su entidad será mayor y más perfecta que la de cualquier accidente ${ }^{9}$. De ahí que, si los accidentes pueden existir por una acción especial de Dios sin su sujeto como sucede en la Eucaristía, con más razón la materia puede, por una acción especial de la voluntad absoluta de Dios, existir sola sin forma alguna ${ }^{10}$.

Por lo dicho vemos las analogías existentes entre la materia de Enrique de Gante y la de Juan Duns Escoto. Quiero, sin embargo, destacar una diferencia importante: la materia de Enrique de Gante tiene esencia y ser propio pero este ser propio no está en acto. Es un extraño ser en potencia, imperfecto y carencial que exige, para lograr su plenitud óntica, el ser en acto recibido de la forma y constituir, con ese ser en acto, el individuo sustancial compuesto. La materia de Duns Escoto, por el contrario, tiene esencia y ser propio en acto, un acto mínimo e imperfecto que le permite ser enormemente receptivo de la forma sustancial que lo perfecciona. La unidad de la sustancia compuesta será, por tanto, entendida de distinta manera por nuestros dos autores. Para Enrique de Gante la unidad de la sustancia compuesta proviene del único ser en acto de dicha sustancia compuesta, a saber, el ser en acto de la forma. Para Duns Escoto, la unidad de la sustancia compuesta proviene de la subordinación del ser en acto mínimo de la materia al ser en acto de la forma que lo perfecciona y lo consolida dentro de la sustancia.

\section{VISIÓN ESCOTISTA DE LA MATERIA PRIMA DE ENRIQUE DE GANTE}

Entremos ahora en el objetivo de este trabajo, a saber, la comprensión que Duns Escoto tiene del concepto de materia prima desarrollado por Enrique de Gante. Sabemos que Duns Escoto conoció y estudió los textos del Doctor sólido. Las citas frecuentes así nos lo confirman. No es raro, por tanto, que cuando intente elaborar su concepto de materia prima, el Doctor Sutil tenga en cuenta lo dicho por Enrique de Gante, autoridad doctrinal importante y, en ese momento, de reconocida ortodoxia. Duns Escoto hace referencia a la doctrina sobre la materia prima del Doctor Sólido en dos ocasiones: a) en Lectura II, dist. 12, q. Única, $\mathrm{n}^{\circ} 45-47$ dentro de la cuestión única en la que se pregunta 'si, en la sustancia generable y corruptible, existe alguna entidad positiva distinta de la forma, llamada materia', b) en Quaestiones in Secundum Librum Sententiarum, cuando se pregunta «si la materia prima por (acción de) alguna potencia puede existir sin la forma» ${ }^{11}$.

8 Ioannes Duns Scotus, Reportata parisiensia, liber II, dist. XII, a. 1, n 17 (Opera Omnia, Edición WaddingVives, Paris, 1893, t. 23, p. 9b): materia habet vere esse extra causam et materia est vere actus, ut distinguitur contra potentiam.

9 Ioannes Duns Scotus, Quaestiones in Secundum Librum Sententiarum, Liber II, dist. XII, q. 2, nº 6 (Opera Omnia, Edición Wadding-Vives, t. 12, p. 578a: dico quod omne esse cujuscumque alterius generis a substantia, scilicet accidentis, est imperfectius quocumque quod est pars substantiae sicut est materia.

10 Ioannes Duns Scotus, Reportata parisiensia, Liber II, dist. 12, q. II, $\mathrm{n}^{\circ}$ 1, contra (Opera Omnia, edición Vives, t. 23, p. 14 a -b): minus videtur accidens posse esse sine subjecto quam materia sine forma, quia subjectum aliquam causalitatem habet respectu accidentis; sed materia in nullo genere causae dependet a forma. Sed accidens per aliquam virtutem poterit esse sine subjecto, igitur et materia sine forma.

11 Ioannes Duns Scotus, Quaestiones in Librum Secundum Sententiarum, Liber II, dist. 12, q. 2 (Opera Omnia, Edición Wadding-Vives, t. 12, p. 574a): Utrum per aliquam potentiam materia possit esse sine forma?... 


\subsection{El texto de la Lectura II, dist. 12, q. Única, $\mathbf{n}^{\circ} \mathbf{4 5 - 4 7}$}

La doctrina de Enrique de Gante es introducida para explicar la dificultad de quienes sostienen que si la materia posee una entidad propia distinta del ser en acto de la forma, no podría la sustancia compuesta constituir un uno per se. Por entidad propia entiende Duns Escoto una esencia propia y un ser en acto propio, aunque ínfimo e imperfecto. La doctrina que Escoto va a atribuir a Enrique de Gante del triple ser, explicará la dificultad de constituir un uno per se subsistente con dos seres en acto. Veamos:

Algunos dicen que la materia tiene un triple ser: un ser lo tiene — si está separada de la forma-y lo recibiría del agente supernatural; el otro es el ser quiditativo «por el que es una cierta capacidad de formas», el que aparece en el XII de la Metafísica: 'la materia prima es una según su sustancia y múltiple según su habilidad'; el tercer ser, apropiado y cierto, se lo comunica la forma «por el que es soporte del compuesto».

Descompongamos el texto en proposiciones de acuerdo al sentido:

1. Algunos dicen que la materia tiene un triple ser: un ser lo tiene — si está separada de la forma-y lo recibiría del agente sobrenatural.

2. El otro ser es el quiditativo, por el que es una cierta capacidad de formas, tal como aparece en el XII de la Metafísica «la materia prima es una según su sustancia y múltiple según su habilidad».

3. El tercer ser, apropiado y cierto, se lo comunica la forma, por el que es soporte del compuesto.

El texto contempla dos posibilidades distintas en las que la materia puede existir: a) de manera natural, en el compuesto sustancial en unión con la forma, b) de manera sobrenatural, separada de la forma, sóla. En ninguno de los dos casos, Duns Escoto descubre el triple ser del que habla Enrique de Gante. En el compuesto sustancial, la materia prima tendría un doble ser, a saber, el ser propio y quiditativo o esencial y el ser recibido de la forma para, ambas, constituir el compuesto o sustancia individual natural. De igual manera, en la hipótesis de que la materia prima fuese creada sola, sin forma, tendría solo dos seres: el propio o esencial por el que es capaz de recibir múltiples formas sustanciales y el ser recibido de manera sobrenatural. Por otra parte, el texto de Enrique de Gante, en el que expone el triple ser que la materia prima posee, es claro, lo que evidencia que la lectura de Duns Escoto no reproduce fielmente la doctrina del Doctor Sólido. Veamos:

Hay que considerar en la materia un triple ser, a saber: ser simplemente y un doble ser algo, a saber, el ser por el que es una cierta capacidad de recibir formas y el ser que es soporte del compuesto. El ser primero, por el que la materia tiene que decirse ser simplemente, lo tiene por medio de una cierta participación en el ser de Dios, en cuanto por la creación es su efecto como los demás, tal como se ha dicho. El segundo ser, por el que la materia es una cierta capacidad, lo tiene por su naturaleza por la que es un id quod est, diferente de la forma. Y hablando de este ser, los seres son diversos de acuerdo a como las esencias son diversas. El ser tercero no lo tiene la materia sino por el hecho de que recibe en sí aquello para lo que de suyo es capaz. De aquí que lo que recibe, le da el ser tal y como eso es la forma, la cual no puede dar sino lo que tiene, el ser que tiene la forma en razón de la naturaleza de su esencia y por medio del que perfecciona la potencia y la capacidad de la materia, lo comunica a la materia y a todo el compuesto ${ }^{12}$.

12 Henricus de Gandavo, Quodlibet I, art. 10, p. 68, vv. 43-56: Est igitur secundum iam dicta in materia considerare triplex esse: esse scilicet simpliciter, et esse aliquid duplex, unum quo est formarum quaedam capacitas, aliud quo est compositi fulcimentum. Esse primum, quo materia habet dici 'ens simpliciter', habet participatione 
Desmembremos este párrafo en proposiciones:

1. Hay que considerar en la materia un triple ser: ser simplemente y un doble ser algo, a saber, el ser por el que es una cierta capacidad de recibir formas y el ser que es soporte del compuesto.

2. El primer ser, por el que la materia se dice ser simplemente, lo tiene por una cierta participación en el ser de Dios, en cuanto es uno de tantos efectos de la creación.

3. El segundo ser, por el que (la materia) es una cierta capacidad, lo tiene por su naturaleza por la que es un id quod est, diferente de la forma. Y este ser lo tienen distinto los seres, de acuerdo a sus distintas esencias.

4. El tercer ser lo tiene la materia por el hecho de recibir aquello (la forma) para lo que de suyo es capaz. Lo que (la materia) recibe, le da el ser tal y como eso es la forma, la cual no puede dar sino lo que tiene en razón de la naturaleza de su esencia y por medio del que perfecciona la potencia y la capacidad de la materia, lo comunica a la materia y al compuesto.

Descubrimos claramente los tres seres que tiene la materia prima: el primer ser es un ser indeterminado, que tiene su origen en la participación que todo ser creado tiene en el ser de Dios, en razón de ser un efecto de la creación. A este ser, común e indeterminado, hay que añadir una doble manera de ser algo que son descritas como el segundo y el tercer ser. La primera manera de ser algo, descrita como el segundo ser de la materia, consiste en una cierta capacidad de recibir formas cuya raíz está justamente en la naturaleza o esencia misma de la materia, diferente de la esencia de la forma. Este segundo ser es distinto de acuerdo a las distintas esencias. Por último, la segunda manera de ser algo, descrita como el tercer ser, lo recibe la materia de la forma; ser en acto que hace que la materia y el compuesto sean tales y por medio del cuál, la forma perfecciona la potencia y la capacidad de la materia.

Este tercer ser, continuará diciendo Enrique de Gante, es el único que la materia tiene en acto y por el que la materia adquiere la existencia actual ${ }^{13}$. Las dos primeras maneras de ser no dan a la materia la existencia actual: la primera, común a todas las criaturas, simplemente hace que la materia participe del ser de Dios, por el hecho de ser uno de tantos efectos de la creación; el segundo le da el ser algo determinado en razón de su esencia. Este segundo ser es el ser que sigue como una sombra a toda esencia y, en concreto, es el ser esencial que sigue siempre a la esencia de la materia y le da la capacidad de recibir formas. Estas dos primeras maneras de ser serán, años más tarde, considerados como seres esenciales que, aún perteneciendo a la realidad, no introducen a la materia en la existencia actual o mundo físico. A la existencia actual del mundo físico, al que pertenecen los supuestos o individuos, entra la materia, perfeccionada en su potencia, sólo por medio del ser en acto recibido de la forma en el compuesto. Por este ser en acto de la forma, la débil entidad de la materia es perfeccionada y se constituye hoc aliquid o sustancia individual que es lo único que verdaderamente existe en el plano de la realidad física. La materia en la sustancia individual compuesta desempeña el papel de soporte (fulcimentum) de la forma sustancial.

La doctrina de Enrique de Gante del triple ser de la materia prima aparece clara en este texto. Duns Escoto, sin embargo, no descubre, en su texto de la Lectura II, el primer ser inde-

quadam a Deo, in quantum per creationem est effectus eius sicut et alia, ut dictum est. Esse secundum, quo materia est capacitas quaedam, habet a sua natura, qua est id quod est, differens a forma. Et loquendo de tali esse, esse sunt diversa quorumcumque essentiae sunt diversae. Esse tertium non habet materia nisi per hoc quod iam capiat in se illud cuius de se capax est. Unde et id quod capit, dat ei tale esse et quia illud forma est, quae non potest dare alteri nisi quod habet, esse igitur quod habet forma ex natura essentiae suae per hoc quod perficit potentiam et capacitatem materiae, communicat materiae et toti composito. Ver el estudio de A. Pérez-Estévez, «La Materia en Enrique de Gante» en Revista Española de Filosofia Medieval, Sofime, Zaragoza, nº 8(2001), pp. 155-175.

13 Ibidem:, Et tale esse (formae) est illud quod materia habet in actu et per quod habet actualem existentiam... 
terminado en razón de ser una criatura y que, en unión del segundo ser o esencial, dispone a la materia prima para recibir el ser en acto de la forma y constituir la sustancia individual compuesta que existe en la naturaleza.

El texto escotista de la Lectura II continúa explicando por qué, para Enrique de Gante, le resulta imposible que el ser sobrenatural que recibe la materia informe se añada al ser en acto de la forma en el compuesto o sustancia individual natural. En efecto, dice:

Dicen que si la materia mantuviese en el compuesto aquel ser que le comunica el agente sobrenatural (si existiese separada) no se haría un uno per se en unión de la forma ni, en ese caso, el compuesto sería un uno per se de manera semejante a como la materia en una cosa artificial no hace un uno per se con la forma artificial; ese ser no lo tiene en el compuesto (aunque pueda tenerlo por la acción de un agente sobrenatural si existe separada) sino que, en el compuesto, posee solo su ser quiditativo y el ser que le comunica la forma. Por eso el compuesto es un uno per se pues tiene solo un ser actual. Así ha de entenderse aquello del II De anima: «no es preciso preguntar si son uno el cuerpo y el alma o la cera y la figura» en donde El Comentador (añade): «al congregado se le dice uno sólo por la unidad existente en la forma» ${ }^{14}$.

Duns Escoto explica por qué no pueden, en la sustancia compuesta natural, encontrarse el ser recibido de manera sobrenatural y el ser de la forma. El compuesto no sería un uno per se. Sin decir claramente qué tipo de ser recibe la materia del agente sobrenatural, simplemente niega la posibilidad de que en la sustancia compuesta puedan darse juntos el ser recibido sobrenaturalmente y el ser de la forma, debido a que no constituirían un uno per se. Sin el ser recibido sobrenaturalmente, la sustancia compuesta de materia prima y forma sustancial constituyen un uno per se, debido, dice, a que tiene un solo ser actual, a saber, el ser en acto de la forma. Se sobreentiende, por tanto, que el ser recibido sobrenaturalmente es otro ser en acto: no puede, con el ser de la forma, constituir un uno per se. Dos seres en acto juntos no constituyen un uno per se. Y lo prueba con el texto de Averroes en el que afirma que «el congregado se dice uno, solo por la unidad existente en la forma» o por tener un solo ser en acto. En todo este texto, Duns Escoto no explica tampoco el triple ser de la materia prima, del que habla Enrique de Gante. Continúa hablando de un doble ser de la materia prima, tanto en el compuesto sustancial como en la hipótesis de una materia prima informe, a saber, el ser esencial o quiditativo y el ser en acto recibido ya sea de la forma en el compuesto, ya sea del agente sobrenatural en la hipótesis de la materia prima informe.

El texto escotista continúa para rechazar, por falsa, la doctrina de Enrique de Gante en la que explica que la materia prima informe necesita, para existir, recibir, como algo añadido, el ser en acto de manera sobrenatural. Ya no es la materia prima sola e informe la que puede existir, sino la materia prima con el ser en acto añadido de manera sobrenatural. Veamos:

Eso no parece ser verdadero, debido a que ningún acto nuevo se produce ni es preciso exigir una nueva acción con respecto al accidente cuando se destruye su sujeto y el mismo se conserva; por eso, nada positivo - absoluto o relativo- se produce en el accidente separado. De igual manera, si la materia pudiese conservarse sin la forma o una vez corrompida la forma, como éstos conceden, no es necesario que un nuevo ser se lo

14 Ioannes Duns Scotus, Lectura II, dist. 12, q. Unica, nº 46 (Opera Omnia, edición Vaticana, t. XIX, pp. 8687): Dicunt igitur quod si materia maneret in composito secundum illud esse quod sibi communicaretur ab agente supernaturali (si esset separata), non faceret unum per se cum forma, nec tunc compositum esset per se unum, sicut nec materia in re artificiali facit unum per se cum forma artificiali; sed illud esse non habet in composito (licet posset habere per actionem agentis supernaturalis, si esset separata), sed habet in composito tantum suum esse quiditativum et esse communicatum sibi a forma; et ideo compositum est per se unum, habens tantum unum esse actuale. Et sic intelligendum est illud II De Anima: «non oportet quaerere si est unum anima et hábeas, sicut nec ceram et figuram»; ubi Commentator: «congregatum non dicitur unum nisi proptr unitatem existentem in forma». 
comunique Dios a ella (la materia) separada. En ese caso, la materia no podría existir per se si no pudiera separarse sin recibir algún ser nuevo, debido a que entonces no podría ser per se sin recibir el ser y el acto; pues con respecto a lo propuesto, no puede (la materia) existir sin ese acto y sin ese ser, así como sin su forma propia. De ahí que si la materia existiese separada, nada nuevo tendría, sino solo la negación de la composición o de su unión con la forma, de manera análoga a como el accidente existe separado de su sujeto $^{15}$.

El rechazo de Duns Escoto a la teoría del de Gante para explicar cómo puede la materia prima existir sin su forma, se debe a que no sería la materia sola, informe, la que podría existir sino la materia prima más el ser en acto añadido por Dios de manera sobrenatural. Para probar la falsedad de esta doctrina, apela a la analogía con lo que sucede en la Eucaristía, cuando los accidentes se quedan existiendo, solos, sin su sujeto que los soporte; de igual manera, la materia prima para poder existir sola, tendría que existir sin ningún ser en acto añadido por Dios de manera sobrenatural. La doctrina de Enrique de Gante, que explica cómo la materia prima puede existir sola, informe, es la prueba de que la materia sola, sin algún ser en acto añadido por la acción de un agente sobrenatural, no puede existir sola. La materia informe podría existir, no por sí misma, sino por el ser en acto añadido que Dios le daría de forma sobrenatural.

Como conclusión de este análisis del texto de la Lectura II, tendríamos que Escoto no alcanza a captar el triple ser que Enrique de Gante atribuye a la materia prima cuando, unida a la forma, constituye una sustancia individual compuesta. Se le escapa siempre el ser indeterminado que la materia prima posee por ser criatura y participar del ser divino. El ser esencial o quiditativo sería un ser pero en potencia. Tampoco comprende bien el ser en acto que, según el Doctor Sólido, Dios daría al ser quiditativo de la materia prima, en la hipótesis de que la hiciera existir sin forma. El ser en acto recibido de Dios, según Duns Escoto, sustituiría al ser en acto formal, para que la materia existiera sóla sin la forma. Enrique de Gante, sin embargo, afirma que la acción divina consistiría en dotar al ser quiditativo de la materia con un ser en acto tan imperfecto que conservaría la tendencia y la disposición esencial a recibir el ser en acto más perfecto de la forma. La materia prima informe, tendría, en consecuencia, una existencia precaria y transitoria, conservando siempre su potencia para recibir el ser en acto perfecto de la forma específica y, unidas, constituir la sustancia compuesta individual. Dios, afirma Enrique de Gante, tendría que hacer que

la materia prima por su naturaleza sea algo en acto subsistente, aunque no en un acto tan perfecto como el que tiene bajo la forma en el compuesto, dado que su acto propio está en potencia hacia el otro acto posterior ${ }^{16}$.

15 Ioannes Duns Scotus, Lectura II, dist. 12, q. Unica, n 47 (Opera Omnia, Edición Vaticana, t. XIX, p. 8788): sed istud non videtur verum, quia nullus actus novus fit, nec actionem novam oportet ponere circa accidens, ex hoc quod destruitur suum subjectum et ipsum conservatur; unde nihil positivum - nec absolutum nec relativumex hoc causatur circa accidens separatum. Eodem modo, si materia posset conservari sine forma vel corrupta forma, sicut ipsi concedunt, non oporteret aliquod esse novum sibi communicari a Deo, sic separantem eam: tunc enim materia non posset esse per se si non posset separari nisi daretur aliquod esse novum, quia tunc non posset esse per se nisi sibi communicaretur esse et actus; et tamen est ad propositum quod non potest esse sine illo actu et sine illo esse, sicut nec sine sua propria forma. Unde si materia esset separata, nihil de novo haberet, sed tantum negationem compositionis vel coniunctionis eius cum forma, sicut si accidens sit separatum a subjecto suo.

16 Henricus de Gandavo, Quodlibet I, q. 10, p. 71, vv. 99-01: Ipsa autem, Deo conservante, quod in ipsa potest creare absque omni actione formae, potest habere a sua natura, quod sit aliquid in actu subsistens, licet non in tam perfecto actu qualem habet in composito sub forma, quia suus actus proprius est in potentia ad actum ulteriorem. 


\title{
1.2. Texto de las Quaestiones in Secundum Librum Sententiarum
}

\author{
Detengámonos ahora a analizar el segundo texto:
}

A lo otro responde un Doctor (Enrique de Gante) que la materia ha de entenderse como poseyendo un triple ser. El primero es el ser suyo que lo tiene debido a que es una esencia distinta de cualquier otra esencia. El otro ser se lo comunica la forma. El tercer ser se lo comunica Dios de manera sobrenatural y este ser que le comunica Dios no lo tiene cuando forma parte del compuesto, pues repugnaría a la unidad del supuesto, pero lo recibiría si Dios la creara sin forma. Pero esto es falso, debido a que esto equivale a decir que la materia, sin algo añadido, no puede existir sin la forma ${ }^{17}$.

El texto intenta reproducir la doctrina de un Doctor (Enrique de Gante) que atribuye a la materia prima un triple ser. El primer ser, es el ser esencial o propio de la esencia de la materia y le corresponde por ser una esencia distinta de cualquier otra esencia. El segundo ser lo recibe de la forma en la sustancia individual compuesta. El tercero lo recibiría de Dios, de manera sobrenatural, sólo en el caso hipotético de que la materia fuese creada sin la forma. Este tercero no lo tiene cuando, con la forma, constituye una sustancia individual compuesta; la razón por la que no puede la materia poseer este tercer ser en el compuesto es porque repugnaría a la unidad del mismo. Es decir, la materia, para Enrique de Gante según esta lectura, tendría sólo dos seres. En efecto, en el compuesto sustancial serían el ser propio esencial que le corresponde por ser una esencia distinta de la forma y el ser en acto recibido de la forma. En la hipótesis de la materia creada sin forma, serían el ser propio esencial y el ser recibido de Dios de manera sobrenatural, ya que no tiene el ser en acto recibido de la forma. ¿Dónde se encuentra el tercer ser? Duns Escoto añade que esta doctrina es falsa pues equivale a decir que la materia, sin algo añadido (el ser recibido inmediatamente de Dios), no puede existir sin la forma. Termina rechazando esta doctrina con una razón de analogía con los accidentes del pan en la Eucaristía: así como los accidentes, una vez transustanciado el pan, no reciben otro ser distinto al que tenían cuando estaba en acto informando el pan sino que se conservan los mismos accidentes, de manera semejante en la hipótesis de que la materia fuese creada sola, sin forma alguna, no se le añadiría otro ser distinto del que tiene cuando existe bajo una forma ${ }^{18}$.

Encontramos repetida la misma lectura, a saber, que tanto en la sustancia natural como en la materia separada de la forma se dan solo dos seres. En la sustancia natural o compuesto se dan el ser quiditativo y el ser comunicado por la forma; en la materia separada, el ser quiditativo y el ser recibido sobrenaturalmente de Dios. El ser sobrenatural, recibido de Dios, no puede encontrarse también en la sustancia compuesta, pues, en ese caso, no podría formarse un uno per se. Tampoco en este texto descubre Duns Escoto, en la sustancia natural, el triple ser del que habla Enrique de Gante.

Como vemos, la doctrina reproducida por Duns Escoto no responde a la doctrina original de Enrique de Gante. El triple ser de la materia se da en toda sustancia individual compuesta,

17 Ioannes Duns Scotus, Quaestiones in Secundum Librum Sententiarum, liber II, dist. 12, q. 2, $\mathrm{n}^{\circ} 9$ (Opera omnia, Edición Wadding-Vives, t. 12, p. 604b): Ad aliud respondet unus Doctor quod materia potest intelligi habere triplex esse. Primo esse suum quod debetur sibi secundum quod est aliqua essentia distincta a quacumque alia. Aliud esse quod communicatur sibi a forma. Tertium esse quod communicatur sibi supernaturaliter a Deo et illud esse communicatum sibi a Deo, non habet quando est in composito, quia repugnaret per se unitati suppositi, sed illud haberet si Deus faceret ipsam sine forma. Sed hoc est falsum quia hoc non est aliud dicere quam materiam sine omni addito non posse esse sine forma.

18 Ioannes Duns Scotus, O.c., liber II, dist. 12, q. 2, n9 (Opera Omnia, edición Wadding-Vives, t. 12, p. 604b): Item sicut accidenti, postquam panis transubstantiatur, non communicatur aliud esse quam habuit, quando fuit acto informans panem, sed idem sibi conservatur (sicut probabitur in quarto libro); ergo similiter in proposito non communicaretur materiae aliud esse ab illo quod habet, dum est sub forma. 
en todo hoc aliquid, en la naturaleza, tal como ha sido creada por Dios. Duns Escoto no habla de ese primer ser indeterminado, que la materia posee por ser criatura de Dios y que le hace participar del ser divino. El segundo, es el ser propio de su esencia, es decir, el ser que sigue a toda esencia por ser distinta de todas las demás esencias. Y el tercero es el ser en acto recibido de la forma, por el que la sustancia individual compuesta se constituye. El triple ser le pertenece a la materia en su natural manera de existir, es decir, unida a la forma en un individuo sustancial. Duns Escoto no descubre este triple ser en la materia real y existente en el mundo natural, tal como quiere Enrique de Gante. La materia real, según la lectura de Duns Escoto, solo poseería dos seres: el esencial y el ser en acto formal.

¿Por qué no reproduce en este texto correctamente la doctrina de Enrique de Gante? Probablemen̂te porque no tenía a mano el texto original y recordaba, en el momento de redactar este texto, confusamente su doctrina. De hecho, va a dar de inmediato otra posible versión de la doctrina del triple ser que Enrique de Gante atribuye a la materia.

$\mathrm{Si}$, por el contrario, entiende que a la materia separada de la forma y sin forma Dios le comunica el ser, no como algún acto, sino como negación del mismo ser comunicado por la forma, es decir, que la materia sea existente per se sin el ser que le comunica la forma, de manera que el ser per se sea una cierta negación del ser comunicado por la forma, dado que en este ser per se, no recibe el ser de la forma. Si, repito, así lo entiende, puede concederse que, a la materia separada, Dios le comunica este tipo de ser o el ser per se negativo, tal como se ha dicho. En consecuencia, puede también decirse que tal ser no le conviene en el compuesto y por la misma razón dicha anteriormente, a saber, porque el compuesto, según esto, no sería uno. Es preciso para la unidad per se del compuesto, que la materia reciba la forma y la soporte y no que a la materia, cuando no está bajo la forma, se le dé un nuevo acto ${ }^{19}$.

Descompongamos párrafo en proposiciones según el sentido:

1. Si por el contrario entiende que a la materia sin forma Dios le comunica el ser, no como algún acto, sino como negación del mismo ser comunicado por la forma,

2. Es decir, si entiende que la materia existe per se sin el ser que recibe de la forma, de manera que el ser per se sea como una negación del ser recibido de la forma,

3 . En ese caso, puede concederse que a la materia sin forma, Dios le da el ser per se negativo en el sentido dicho.

4. En consecuencia, puede decirse que tal ser (per se) recibido inmediatamente de Dios, no le conviene en el compuesto y por la misma razón antes dicha: porque el compuesto no sería uno.

5. Es preciso para la unidad per se del compuesto que la materia reciba la forma y la soporte y no que a la materia, cuando no está bajo la forma, se le dé un nuevo acto.

En esta segunda lectura, aparece Dios dándole inmediatamente a la materia sin forma el ser subsistente por el que existe per se. Un ser que no es acto alguno y, en consecuencia, no es un ser en acto semejante al recibido de la forma en el compuesto individual sino más bien un ser que es la negación del ser en acto formal. En ese caso, dice, puede concederse que a la ma-

19 Ioannes Duns Scotus, Quaestiones in Secundum Librum Sententiarum, liber II, dist. 12, q. 2, $\mathrm{n}^{\circ} 9$ (Opera omnia, Edición Wadding-Vices, t. 12, p. 604b):: Si vero intelligit sic quod materiae separatae a forma, et sine forma, communicatur esse a Deo, non actus aliquis sed negatio ipsius esse communicati a forma, id est, quod materia sit per se existens sine esse sibi communicato a forma, ita quod per se esse sit quaedam negatio talis esse communicati a forma, quia in isto esse per se, non recipit esse a forma. Si, inquam, sic intelligit, potest concedi quod materiae separatae communicatur a Deo hujusmodi esse vel per se esse negative, ut dictum est, et consequenter etiam posset dici quod tale esse non convenit sibi in composito et eadem ratione sicut supra dictum est, quia compositum, secundum hoc, non esset unum. Oportet enim ad unitatem per se compositi, materiam recipere formam et subesse formae, non quod tunc materiae detur aliqua actualitas nova, quando non est sub forma. 
teria, sin forma, Dios le dé, inmediatamente y de manera sobrenatural, el ser por el que exista per se, sin necesidad del ser en acto formal.

Detengámonos a analizar este texto que representa una segunda lectura de Escoto sobre la doctrina de la materia prima de Enrique de Gante. Lo menos que podemos decir de este texto, es que es confuso, ambiguo y tampoco reproduce la doctrina del Doctor Sólido. Nada corrige de lo dicho sobre la materia física y existente, a la que solo le había atribuido un doble ser: el ser esencial y el ser en acto formal. Enrique de Gante, como ya hemos dicho, le atribuye a la materia física existente un triple ser: el ser indeterminado de la criatura, el ser más determinado de la esencia y el ser en acto que recibe de la forma en el compuesto. Esta nueva lectura solo intenta corregir lo dicho sobre la hipotética posibilidad de que la materia sea creada sin forma alguna. Ahora afirma que, según la doctrina de Enrique de Gante, esta supuesta materia, creada sin forma, recibiría de Dios inmediatamente el ser, no como algún acto, pero subsistente per se. Un ser subsistente per se, que es la negación del ser en acto que, de manera natural, recibe de la forma en la sustancia compuesta individual. Es decir, Dios le daría a la materia informe el existir per se, sin el ser en acto formal. No dice que Dios dé directamente a la materia prima el ser en acto recibido de la forma en la naturaleza, sino que «Dios le comunica el ser, no como algún acto, sino como negación del mismo ser comunicado por la forma». Parece Duns Escoto entender que, según Enrique de Gante, Dios, en la hipótesis de que la creara sola sin forma, daría a la materia prima el ser subsistente per se, un ser que no es un ser en acto análogo al recibido de la forma. La materia prima existiría sola, de manera sobrenatural, sin el ser en acto que, de manera natural, recibe de la forma en el compuesto sustancial. Esa materia prima existiría milagrosamente sola sin ser en acto, lo que parece contradictorio.

Comparemos este segundo texto dado por Duns Escoto con el original de Enrique de Gante. Éste afirma que Dios puede crear, de manera sobrenatural, la materia prima sola sin forma, pero, en ese caso, Dios haría que:

la materia prima por su naturaleza sea algo en acto subsistente, aunque no en un acto tan perfecto como el que tiene bajo la forma en el compuesto, dado que su acto propio está en potencia hacia el otro acto posterior ${ }^{20}$.

Dios, en el supuesto de que creara la materia prima sola sin forma, haría que fuese por su naturaleza algo en acto subsistente. Dios haría que el ser esencial de la materia prima, que consiste en la capacidad de recibir formas sustanciales, fuese en acto, pero no en un acto tan perfecto como el que tiene bajo la forma en el compuesto. Es decir, el ser esencial de la materia prima sería, en el supuesto de que fuese creada sola sin forma, algo en acto imperfecto que conservaría la potencia y la tendencia esencial a recibir el ser en acto de la forma en el compuesto. Esta hipotética materia prima tendría sólo dos seres: el ser indeterminado de toda criatura y el ser propio esencial que vendría a ser la mezcla de un ser en acto subsistente imperfecto y de su esencial potencia de recibir formas sustanciales. Su tendencia a recibir el ser en acto formal permanecería en esa materia informe o como dice textualmente «su acto propio está en potencia hacia el otro acto posterior».

Como vemos, tanto la primera como la segunda lectura que hace de la doctrina del Doctor Sólido son incorrectas e incompletas. Nunca descubrió ese primer ser indeterminado, por ser criatura de Dios, con que Enrique de Gante dota a toda materia prima existente, incluida la hipotética materia prima, sin forma, que, según el Doctor sólido, podría ser creada por una

20 Henricus de Gandavo, Quodlibet I, q. 10, p. 71, vv. 99-01: Ipsa autem, Deo conservante, quod in ipsa potest creare absque omni actione formae, potest habere a sua natura, quod sit aliquid in actu subsistens, licet non in tam perfecto actu qualem habet in composito sub forma, quia suus actus proprius est in potentia ad actum ulteriorem. 
acción milagrosa de Dios. Duns Escoto, aunque afirma que Enrique de Gante atribuye un triple ser a la materia, no descubre ese triple ser, debido a que se le escapa el ser indeterminado primero por el que es una criatura. Tampoco Duns Escoto es capaz de descubrir el ser en acto imperfecto que la hipotética materia prima informe recibe directamente de Dios y que le da la subsistencia per se. En la segunda lectura, el Doctor sutil le hace decir a Enrique de Gante que la materia prima informe existiría o subsistiría per se, sin poseer acto alguno, lo que sería evidentemente contradictorio.

Hemos de llamar la atención sobre el cambio que encontramos en el texto de Duns Escoto en Lectura II, dist. 12, q. Unica, ${ }^{\circ} 47$ (Opera Omnia, Edición Vaticana, t. XIX, p. 88), en el que se afirma que la doctrina de Enrique de Gante es falsa, debido a que la materia por sí misma no puede subsistir y, para subsistir sola, necesita recibir de Dios

«algún ser nuevo, pues no podría ser per se sin recibir ser y acto; y tiene que ver con lo propuesto que no puede ser sin ese acto y sin ese ser así como sin su forma propia» ${ }^{21}$.

En este texto la materia separada de la forma, en la lectura que hace Duns Escoto, recibe de Dios un nuevo ser y un nuevo acto para que pueda subsistir o existir sola. Tampoco aquí reproduce con exactitud el pensamiento del Doctor Sólido. Para Enrique de Gante, como ya hemos analizado, Dios hace que la materia prima informe, por su naturaleza, sea algo en acto subsistente. Sabemos que la materia prima posee un ser esencial que la capacita para recibir formas sustanciales. Dios fortalece, de manera sobrenatural, ese ser esencial —que no es actoy lo convierte en algo en acto subsistente, capaz de existir sola sin forma alguna. Esta doctrina, repite Duns Escoto, es falsa, porque es la prueba de que la materia con su propio ser esencial y su capacidad de recibir formas sustanciales, sin el acto añadido por Dios, no puede existir sola. Para existir requiere de algún ser en acto, ya sea dado sobrenaturalmente por Dios o dado por la forma en la sustancia compuesta natural. Nada dice Duns Escoto de que este ser en acto recibido sobrenaturalmente de Dios, es un ser en acto imperfecto que no elimina la tendencia natural que la materia prima encierra, para recibir el acto más perfecto de la forma. Por eso, para Enrique de Gante, la existencia de la materia informe será siempre una existencia imperfecta y transitoria, debido a que continúa exigiendo el ser en acto posterior y más perfecto de la forma sustancial.

Es de advertir que, para Enrique de Gante, los dos primeros seres de toda sustancia individual compuesta — el ser indeterminado por ser criatura y el ser esencial— son seres reales aunque no posean la consistencia óntica suficiente para existir sin la compañía del ser en acto de la forma. Es decir, son reales sólo en compañía del ser en acto de la forma en un compuesto individual, que es lo único que existe. En la hipótesis de que la materia prima fuese creada sola sin forma por una acción sobrenatural de Dios, el ser esencial, de suyo incapaz de existir sin el ser en acto de la forma, recibiría de Dios un ser en acto imperfecto que le daría la subsistencia per se y existiría sola. Esa existencia, sin embargo, no sería una existencia tan completa y perfecta como la recibida del ser en acto de la forma, sino imperfecta y, probablemente, transitoria. Su capacidad de recibir el ser en acto de la forma, que constituye la esencia, continuaría en la materia prima, existiendo sola por acción directa de Dios. Esa materia, sola sin forma, sería una materia que existiría de manera sobrenatural con su imperfecto ser en acto, pero sin alcanzar la plenitud del ser en acto formal. Su capacidad y tendencia a recibir de la forma el ser en acto, se completaría solo cuando recibiera el ser en acto de la forma y constituyeran ambas una sustancia compuesta individual.

21 Ioannes Duns Sctous, Lectura II, dist. 12, q. Unica, $\mathrm{n}^{\circ} 47$ (Opera Omnia, Edición Vaticana, t. XIX, p. 86): Tunc enim materia non posset esse per se si non posset separari nisi daretur aliquod esse novum, quia tunc non posset esse per se nisi sibi communicaretur esse et actus; et tamen est ad propositum quod non potest esse sine illo actu et sine illo esse, sicut nec sine sua propria forma. 
.La materia prima, en el hipotético caso de que exista sola sin forma, existiría sin el ser en acto formal pero con un ser en acto imperfecto dado por Dios de manera sobrenatural. Es decir, recibiría de manera sobrenatural un imperfecto ser en acto directamente de Dios, de acuerdo al principio de que Dios puede dar directa e inmediatamente lo que da, de forma mediata, a través de una causa segunda. En este caso, Dios puede dar el ser en acto directa e inmediatamente que, de manera natural, la materia prima recibe de la forma en la sustancia individual, pero sin eliminar la tendencia natural de la misma a recibir el ser en acto formal. El ser en acto recibido directamente de Dios, de manera milagrosa, es un ser en acto imperfecto e incompleto que no elimina la tendencia natural de la materia a recibir la plenitud del ser en acto formal. Enrique de Gante, tiene en cuenta para esta afirmación, el hecho milagroso de la Eucaristía en el que los accidentes de pan o de vino se conservan existiendo, después de la consagración, sin el sujeto propio que los sostenga. Si el accidente que posee una entidad menor que la materia prima puede existir solo, sin el sujeto que los soporta, con mayor razón la materia prima puede existir sola, sin la forma que le da el ser en acto en la sustancia.

\section{CONCLUSIÓN}

Como ya hemos señalado, Duns Escoto no comprende la doctrina del triple ser de Enrique de Gante. No alcanza a descubrir el ser indeterminado que la materia prima posee como criatura de Dios. Sí entiende que el ser en potencia que acompaña a la esencia de la materia prima, que le es propio y la hace distinta de cualquier otra esencia, no posee la consistencia óntica suficiente como para que pueda, incluso de manera milagrosa, existir sola sin forma alguna. Por eso, ese ser esencial y propio de la materia prima tendrá que ser consolidado por Dios de manera sobrenatural. Enrique de Gante dice expresamente que Dios hará que la materia prima por su naturaleza sea algo en acto subsistente, es decir, hace que la materia prima con su segundo ser esencial y propio, que le da la capacidad de recibir el ser en acto formal, se convierta en algo en acto subsistente. Un algo en acto subsistente tan imperfecto que continúa tendiendo al acto de ser formal por el que alcanzaría la existencia plena en una sustancia compuesta individual. Duns Escoto no alcanza a percibir ese imperfecto ser en acto que la materia prima recibe inmediatamente de Dios, para que pueda existir sola sin forma. Con su segunda lectura le hace decir a Enrique de Gante que «la materia prima, de manera sobrenatural, existe per se sin ser en acto", lo que parece contradictorio. Si existir equivale a ser en acto, tendríamos que la materia prima existiría (sería en acto) sobrenaturalmente sin recibir el ser en acto ni de Dios ni de la forma.

Me parece importante señalar que Tomás de Aquino, Enrique de Gante y Juan Duns Escoto significan tres jalones importantes en la evolución del concepto de materia prima en la segunda mitad del siglo XIII. Para Tomás de Aquino la materia prima, en su esencia, es un noente que puede ser, es pura potencia para recibir el acto de ser formal. Existe sólo en la sustancia compuesta individual, una vez recibido el ser en acto de la forma. Recordemos que, para el Aquinate, se dan dos tipos de actos en las sustancias existentes: el acto formal, por el que se constituye la sustancia y el acto existencial por el que la sustancia existe fuera de sus causas. En las sustancias compuestas, el acto formal hace que la materia, unida a la forma, constituyan la sustancia y el acto de existir recibido de Dios, hace que la sustancia exista fuera de sus causas. La materia prima tomista se ve doblemente alejada del ser en acto por una doble potencia: es potencia con respecto al acto de la forma para constituir, ambas juntas, la sustancia compuesta y, unida a la forma en la sustancia, es potencia para recibir de Dios el acto de existir. La materia prima tomista queda reducida a una muy borrosa sombra potencial de ser: es un no-ente que puede ser. La materia prima tomista posee una entidad incluso menor que el accidente. De ahí que el accidente pueda, de manera sobrenatural, existir solo sin un sujeto que lo sostenga, como sucede en la Eucaristía, mientras que es imposible a la materia prima 
existir sola sin forma. Afirmar que la materia prima existe equivale a decir que un no-ente es, es decir, es absolutamente imposible por contradictorio.

Ea materia prima de Enrique de Gante, en su esencia, es algo y posee un ser propio, distinto del ser de la forma, que la capacita para recibir el ser en acto de la forma y constituir, ambas juntas, una sustancia compuesta. Es más, la materia prima existente en la sustancia compuesta posee un triple ser: el ser indeterminado por ser criatura de Dios, el ser esencial y propio que le da la capacidad de recibir formas sustanciales y el ser en acto recibido de la forma. Pero tengamos en cuenta que el ser esencial y propio de la materia prima es un ser con capacidad o potencia para recibir el ser en acto de la forma sustancial. En la sustancia compuesta el único ser en acto es el ser de la forma, causa de la unidad sustancial. La materia prima de Enrique de Gante ha crecido en relación a la de Tomás de Aquino, es un algo, un ente con la capacidad o potencia de recibir el ser en acto de la forma. Posee una realidad, pero no alcanza a tener la consistencia óntica que le permita existir, sola, en el mundo natural creado por Dios. Existe de manera natural solo en la sustancia compuesta unida a la forma y, después de haber recibido de ella el ser en acto sustancial. Para que pueda existir sola, sin forma, es preciso una acción sobrenatural de Dios que consolide ese ser en potencia que le es propio y lo convierta en un ser en acto imperfecto que no elimine su esencial capacidad de recibir el ser en acto formal. Su ser propio es un ser sustancial o per se y, por tanto, superior al ser de cualquier accidente que es siempre ser en otro.

Finalmente la materia prima de Duns Escoto, en su esencia, es un ente y, por tanto, posee un ser en acto propio, pero imperfecto. El ser en acto propio y esencial coloca a la materia prima fuera de sus causas, pero, por ser un acto imperfecto le da la capacidad o potencia de recibir el ser en acto perfecto de la forma, con la que constituye la sustancia compuesta existente. El ser en acto de la materia prima es tan imperfecto que necesita ser perfeccionado y completado por el ser en acto de la forma y, juntas, constituir una sustancia compuesta. Sin el acto de ser formal que la perfecciona, la materia prima no puede existir en el mundo natural creado. El ser en acto propio y esencial de la materia prima es un ser en acto sustancial, lo que hace que su entidad sea mayor que la entidad de cualquier accidente. Por eso, si Dios puede hacer y hace que los accidentes existan solos sin el sujeto propio que los soporte, como sucede de manera milagrosa en la Eucaristía, con más razón la materia prima podría existir sola sin forma alguna, si la potencia absoluta de Dios así lo decidiese.

Estos tres autores - Tomás de Aquino, Enrique de Gante y Duns Escoto- representan momentos importantes en el crecimiento del concepto de materia prima en la segunda mitad del siglo XIII: de ser un no-ente que puede ser en Tomás de Aquino pasa a ser algo en potencia para Enrique de Gante y a poseer un ser-en-acto imperfecto en Duns Escoto. En los tres necesita del ser en acto de la forma para constituir una sustancia individual y existir en la naturaleza. Para Tomás de Aquino es absolutamente imposible, por contradictorio, que la materia exista sola, sin forma. Para Enrique de Gante la materia prima puede existir sola pero por medio de una acción milagrosa de Dios que fortalezca el ser esencial y lo dote de un ser-enacto imperfecto que no la prive de su tendencia a recibir el ser-en-acto de la forma sustancial. Para Duns Escoto, la materia prima puede existir sola, sin forma, si la potencia absoluta de Dios lo decreta.

Antonio Pérez-Estevez aperezestevez@yahoo.es 